\title{
Quasi-half-cycle pulses of light from a tapered undulator
}

\begin{abstract}
V. A. Goryashko*
Department of Physics and Astronomy, Uppsala University, Lägerhyddsvägen 1, Uppsala 75120, Sweden (Received 27 September 2016; revised manuscript received 7 July 2017; published 15 August 2017)

Strong-field few-cycle terahertz $(\mathrm{THz})$ pulses are an invaluable tool for engineering highly nonequilibrium states of matter. A scheme is proposed to generate quasi-half-cycle $\mathrm{GV} / \mathrm{m}$-scale $\mathrm{THz}$ pulses with a multikilohertz repetition rate. It makes use of coherent spontaneous emission from a prebunched electron beam traversing an optimally tapered undulator. The scheme is the further development of the slippage control in free-electron lasers [T. Tanaka, Phys. Rev. Lett. 114, 044801 (2015)]. An explicit condition for the spectral amplitude of undulator radiation and a phase condition for the electron density distribution, required for the generation of desired pulses, are presented. The amplitude condition is met by proper undulator tapering, and a generic optimal undulator profile is found analytically. In order to meet the phase condition, the distance between the adjacent bunches is varied according to the instantaneous resonant undulator wavelength. A $3 \mathrm{D}$ analytical theory is complemented by a detailed numerical study based on a direct solution to the 3D wave equation.
\end{abstract}

DOI: 10.1103/PhysRevAccelBeams.20.080703

\section{INTRODUCTION}

Quasi-half-cycle pulses of light [1-3] are of interest from the fundamental point of view, as they clearly reveal the dispersive nature of spatially structured light in free space [4] manifested as a temporal reshaping of pulses as they propagate through the focus [5]. Such pulses are also of importance for various applications thanks to the preferential direction of their electric fields. For example, quasihalf-cycle pulses of terahertz $(\mathrm{THz})$ and IR light allow the engineering of new highly nonequilibrium states of matter [6] such as light-induced superconductivity $[7,8]$.

Crucial progress in the emerging field of the generation of strong-field few-cycle $\mathrm{THz}$ light has been made during the past decade thanks to the advance of laser-based $\mathrm{THz}$ sources [9-11] based on the optical rectification of short intense optical pulses in a crystal such as lithium niobate. Nevertheless, the radiation spectrum of laser-based sources is limited to the frequencies below $5 \mathrm{THz}$, and the repetition rate of strong-field pulses is some Hz. Another method of generation of quasi-half-cycle $\mathrm{THz}$ pulses is transition radiation from high-energy charged particles traversing a metallic foil $[12,13]$, but it requires multi-GeV electron bunches of a few $\mathrm{nC}$ charge. To boost the emitted energy by an order of magnitude and lower the required bunch energy, a multifoil cone radiator [14] was suggested. However, the radiation spectrum produced by electron bunches from a

\footnotetext{
*vitaliy.goryashko@physics.uu.se; vitgor06@gmail.com
}

Published by the American Physical Society under the terms of the Creative Commons Attribution 4.0 International license. Further distribution of this work must maintain attribution to the author(s) and the published article's title, journal citation, and DOI. small-scale accelerator is limited to a few $\mathrm{THz}$, and a high repetition rate is a challenge because of the heat load on the radiator.

Here, I present an approach for the generation of intense quasi-half-cycle $\mathrm{THz}$ pulses via coherent spontaneous emission from a prebunched electron beam traversing an optimally tapered undulator. This approach opens a way to high repetition rates up to a $\mathrm{MHz}$ level with a superconducting accelerator, which is very attractive for highrepetition rate pump-probe experiments at x-ray light sources such as the European XFEL and Linac-based Coherent Light Source-II. The idea of the presented approach can be thought of as a modification and more quantitative study of Tanaka's scheme $[15,16]$, though it originates from the independent development of a $\mathrm{THz}$ light source at Uppsala University.

The basic idea of the generation of quasi-half-cycle $\mathrm{THz}$ pulses is some kind of mode locking of frequency-chirped coherent spontaneous radiation from electron bunches. Consider a short electron bunch passing through a magnetic field of an undulator. In the periodic undulator field, the bunch emits a sinusoidal impulse with the period dependent on the undulator field. Now, if the undulator field changes along the undulator, then a frequency-chirped impulse is generated. The impulses emitted by two consecutive bunches can interfere constructively at some points if the separation between the bunches is equal to one of the periods of the chirped sinusoidal impulse. By employing a train of bunches with the separation between the adjacent bunches equal to the corresponding period of the frequency-chirped impulse, a few-cycle pulse can be generated. In this paper, we will refer to the radiation from a short electron bunch as an impulse, whereas the term pulse is reserved for the superposition of impulses. 


\section{FROM A FREQUENCY-CHIRPED IMPULSE TO A QUASI-HALF-CYCLE PULSE}

We wish to generate quasi-half-cycle pulses of the form

$$
f(t)=\left(1-\frac{t^{2}}{\sigma_{t}^{2}}\right) \mathrm{e}^{-t^{2} / 2 \sigma_{t}^{2}},
$$

whose Fourier transform reads

$$
f_{\omega}=\sqrt{2 / \pi} \sigma_{t}^{3} \omega^{2} \mathrm{e}^{-\sigma_{t}^{2} \omega^{2} / 2}
$$

Let us see how this pulse can be connected to a frequencychirped impulse generated by the test electron passing though a tapered undulator. Figure 1 depicts some Fourier components of the quasi-half-cycle [Fig. 1(b)] and frequency-chirped [Fig. 1(d)] pulses having the same spectral density distribution. When all the Fourier components are in phase, the quasi-half-cycle pulse is formed. A nonlinear phase shift of the Fourier components results in the frequency-chirped pulse. Hence, in order to generate the desired pulse with the tapered undulator, the spectral undulator distribution must be identical to that of the desired pulse and the electrons must be delayed (positioned) with respect to each other such that the nonlinear phase shift along the chirped undulator impulse is compensated for.

In the spirit of Tanaka's analysis [15], the generation of few-cycle pulses can quantitatively be formulated as

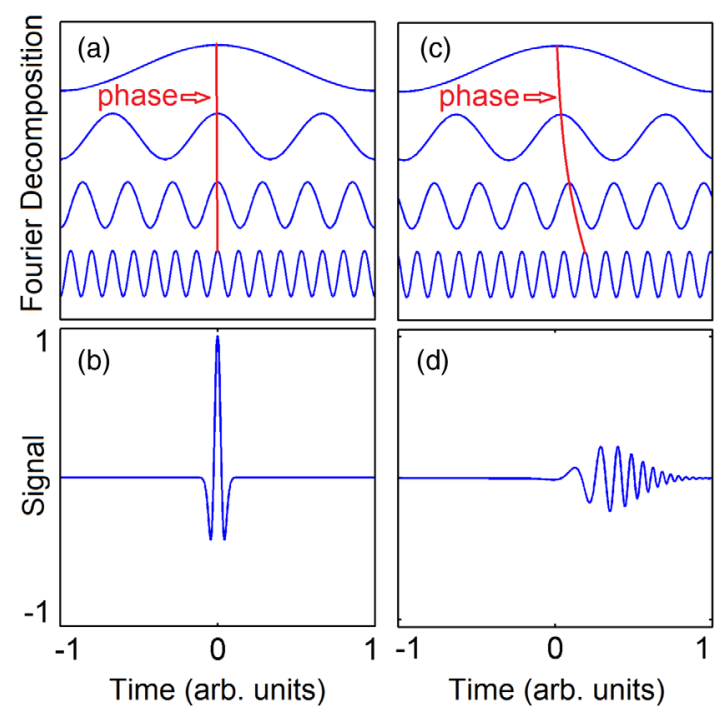

FIG. 1. Schematic illustration of the Fourier decompositions (a) and (c) of the quasi-half-cycle (b) and frequency-chirped (d) pulses, respectively. The quasi-half-cycle pulse is formed by the superposition of in-phase sinusoidal components, whereas the frequency-chirped pulse is composed of the sinusoidals with a nonlinear phase shift in between them. The amplitudes of the Fourier components are not to scale.

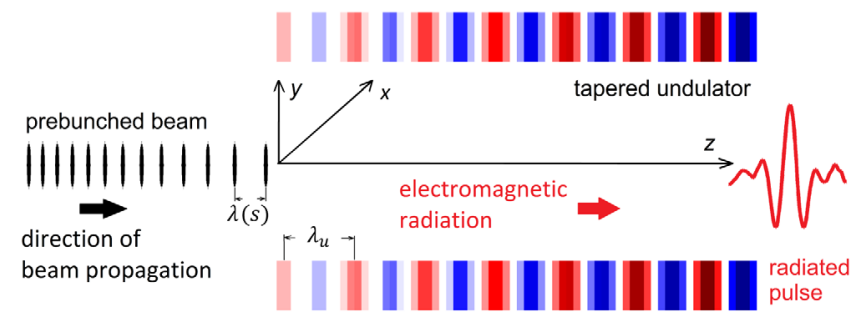

FIG. 2. Schematic of a tapered undulator and a prebunched beam. The distance between the bunches $s$ changes as the radiation wavelength along the undulator impulse $\lambda$.

follows. The total electric field $E(t)$ produced by individual electrons is given by the superposition $E(t)=$ $\int E_{0} p\left(t-t_{0}\right) n_{0} g\left(t_{0}\right) d t_{0}$, where $E_{0}$ and $p(t)$ are the amplitude and profile, respectively, of the undulator impulse; $n_{0}$ and $g(t)$ are the density and profile, respectively, of the electron bunch. Then, the spectral complex amplitude of $E(t)$ is given by

$$
E_{\omega}=E_{0} n_{0} \int p_{\omega} g\left(t_{0}\right) e^{i \omega t_{0}} d t_{0}=E_{0} n_{0} p_{\omega} g_{\omega} .
$$

In order to generate the desired pulse, the amplitude and phase conditions must be met: $\left|g_{\omega} p_{\omega}\right|=f_{\omega}$ and $\arg \left(g_{\omega}\right)=$ $-\arg \left(p_{\omega}\right)$. In the particular case of $g_{\omega}=p_{\omega}^{*}$, as in Ref. [15], the electron density distribution $g(t)$ is a timereversed replica of the chirped undulator impulse $p(t)$.

In this paper, we consider a tapered undulator driven by a train of short bunches with varying spacing in between. First, I derive a general expression for the radiation spectrum of a strongly tapered undulator and then find the optimal undulator profile from the amplitude condition. The phase condition is met by positioning the electron bunches at the distances equal to the radiation wavelength of the undulator impulse. An analytical analysis is followed by a 3D numerical solution to the wave equation with an excitation source in the form of a single bunch and prebunched beam. The geometry of the problem in shown in Fig. 2.

\section{THE MODEL}

We turn to the derivation of the general expression for the field emitted by a short electron bunch traversing a planar tapered undulator, whose symmetry plane is lying in the $x z$ plane with $x$ and $z$ being the transverse (horizontal) and longitudinal coordinates, respectively. The undulator is assumed to be wide in the $x$ direction such that the magnetic field depends only on the transverse (vertical) coordinate $y$ and $z$. Let the magnetic field be the product of a sinusoidal carrier $\sin k_{u} z$ and a slow-varying envelope $f_{u}(z)$. The corresponding vector potential reads

$$
A_{x}^{u}=A_{0} f_{u}(z) \sin k_{u} z \cosh k_{u} y
$$


and satisfies the wave equation under the assumption $\lambda_{u} \mathrm{~d} \ln f_{u} / \mathrm{d} z \ll 1$. Here, $\lambda_{u}$ and $k_{u}$ are the period and wave number, respectively, of the periodic component of the magnetic field. The undulator comprises $N_{u}$ periods, $L_{u}=N_{u} \lambda_{u}$.

Spontaneous undulator radiation is dictated by the trajectories of the electrons; therefore, we now briefly examine them for completeness. We choose $z$ as an independent variable. Since the vector potential is independent of $x$, the corresponding component of the canonical momentum is preserved so that the $x$ component of the electron's velocity normalized to the speed of light $c$ reads

$$
\beta_{x}=\frac{\mathcal{K}}{\gamma} f_{u}(z) \sin k_{u} z \cosh k_{u} y .
$$

Here, $\gamma$ is the electron's energy measured in units of the rest mass, and $\mathcal{K}=|e| A_{0} / m_{e} c^{2}$ is the undulator parameter with $e(<0)$ and $m_{e}$ being the charge and mass of the electron, respectively. A low-emittance electron bunch is considered; hence, the initial spread in velocities is ignored. Motion in the $y$ direction for small deviations is described by the harmonic oscillator equation with a spatial period of $\lambda_{\beta}=\sqrt{2} \gamma \lambda_{u} / \mathcal{K} f_{u}(z)$. The oscillations in $y$-referred to as betatron oscillations - occur on a spatial scale much longer than the undulator period. In what follows, we neglect them assuming that the betatron phase shift is small $\int_{0}^{L_{u}} \mathrm{~d} z^{\prime} / \lambda_{\beta}\left(z^{\prime}\right) \ll 1$, which is valid for a short undulator, i.e. $N_{u} \ll 2 \sqrt{2} \gamma / \mathcal{K}$.

The electron's energy is preserved in a magnetic field, so for a relativistic electron $(\gamma \gg 1)$ the longitudinal velocity and arrival time to position $z$ read

$$
\begin{aligned}
\beta_{z} \approx 1 & -\frac{1}{2 \gamma^{2}}-\frac{\beta_{x}^{2}}{2}=1-\frac{1+\mathcal{K}^{2} f_{u}^{2}(z) \sin ^{2} k_{u} z}{2 \gamma^{2}}, \\
c t_{e} \approx & c t_{0}+\int_{0}^{z} \frac{\mathrm{d} z^{\prime}}{\beta_{z}\left(z^{\prime}\right)} \approx c t_{0}+\left(1+\frac{1}{2 \gamma^{2}}\right) z \\
& +\frac{\mathcal{K}^{2}}{2 \gamma^{2}}\left(\frac{1}{2} \int_{0}^{z} f_{u}^{2}\left(z^{\prime}\right) \mathrm{d} z^{\prime}-f_{u}^{2}(z) \frac{\sin 2 k_{u} z}{4 k_{u}}\right) .
\end{aligned}
$$

Here, $t_{0}$ is the injection time of the test electron. In order to obtain the last two terms in Eq. (6), we used the integration by parts of the term $f_{u}^{2}(z) \sin ^{2} k_{u} z$ appearing in $t_{e}(z)$ and neglected high-order harmonics. Our result (6) is similar to that arising in the studies of Thomson scattering of laser pulses on electron bunches $[17,18]$.

Consider the radiation field copropagating with the electron bunch

$$
E(\vec{r}, t)=\operatorname{Re} \int_{0}^{\infty} E_{\omega}(z) \mathrm{e}^{-i \omega(t-z / c)} \mathrm{d} \omega .
$$

The dominant component of the radiated field is created by the transverse current density $j_{x}$, since the velocity of undulations in the $x$ direction is much larger than that in other directions. Hence, only $E_{x}$ is considered. Assuming that the rate of energy transfer from the electron bunch to the radiation field is low-i.e. $E_{\omega}(z)$ is a slow function of $z$-we may use the paraxial approximation so that the complex Fourier amplitude satisfies the equation

$$
\left(\nabla_{\perp}^{2}+\frac{2 i \omega}{c} \frac{\partial}{\partial z}\right) E_{\omega}=-i \omega \frac{4 \pi}{c^{2}} j_{\omega} .
$$

Here, $j_{\omega}$ is the Fourier transform of the $x$ component of the current density. It reads

$$
j_{\omega}=\frac{Q}{2 \pi \sigma_{b}^{2}} \frac{v_{x}}{v_{z}} F \exp \left[-\frac{r^{2}}{2 \sigma_{b}^{2}}\right] \exp \left[i \omega t_{e}(z)\right],
$$

where $F=\exp \left(-\omega^{2} \sigma_{T}^{2} / 2\right)$ is the bunch form factor, $\sigma_{T}$ and $\sigma_{b}$ are the rms bunch duration and transverse size, respectively, $Q$ is the total charge, and $v_{x}$ and $v_{z}$ are the $x$ and $z$ components of the velocity, respectively.

A solution to (8) is given by the convolution of (9) with the Green function of (8), which is [19]

$$
G=\frac{1}{4 \pi\left(z-z^{\prime}\right)} \exp \left[\frac{i \omega\left|\vec{r}_{\perp}-\vec{r}_{\perp}^{\prime}\right|^{2}}{2 c\left(z-z^{\prime}\right)}\right]
$$

In the approximation of small undulator oscillations compared to the transverse size of the electron bunch, the spectral complex amplitude $E_{\omega}$ at the end of the undulator turns out to be

$E_{\omega}=E_{\omega}^{0} \int_{0}^{L_{u}} g\left(z^{\prime}\right) f_{u}\left(z^{\prime}\right) \exp \left[\frac{-r^{2}}{w^{2}\left(z^{\prime}\right)}+i \Psi\left(z^{\prime}\right)\right] \mathrm{d} z^{\prime}$,

where the amplitude and phase factors read

$$
E_{\omega}^{0}=-\frac{Q \mathcal{K} F(\omega)}{\pi \sigma_{b}^{2} \gamma c}, \quad \Psi(z)=\frac{k r^{2}}{2 R(z)}-\phi(z)+\psi(z)
$$

and $g(z) f_{u}(z)$ is the local envelope with $g(z)$ given by

$$
g\left(z^{\prime}\right)=\frac{J J\left(z^{\prime}\right)}{\sqrt{1+\left(L_{u}-z^{\prime}\right)^{2} / z_{R}^{2}}} .
$$

Here, $z_{R}=k \sigma_{b}^{2}$ is the effective Rayleigh length, $k=\omega / c$, and $J J$ stands for the $J J$ factor [20] responsible for longitudinal wiggling of electrons

$$
J J=J_{0}(A)-J_{1}(A), \quad A=\frac{\mathcal{K}^{2} f_{u}^{2}(z)}{4 \xi} .
$$

The waist, Gouy phase shift, radius of curvature, and ponderomotive phase are 


$$
\begin{aligned}
w^{2} & =2 \sigma_{b}^{2}\left[1+\frac{\left(L_{u}-z\right)^{2}}{z_{R}^{2}}\right], \quad \phi=\arctan \left[\frac{L_{u}-z}{z_{R}}\right], \\
R & =\left(L_{u}-z\right)\left[1+\frac{z_{R}^{2}}{\left(L_{u}-z\right)^{2}}\right], \\
\psi & =\frac{2 \pi}{\lambda_{u} \xi}\left[z(1-\xi)+\frac{\mathcal{K}^{2}}{2} \int_{0}^{z} f_{u}^{2}\left(z^{\prime}\right) \mathrm{d} z^{\prime}\right] .
\end{aligned}
$$

We introduced a normalized wavelength $\xi=\lambda / \lambda_{0}$ with $\lambda_{0}=\lambda_{u} / 2 \gamma^{2}$ being the Doppler-upshifted period of the undulator field. The approximation of small undulator oscillations can be quantitatively written as $\pi N_{u} N_{F} \gg 1$, where $N_{F}=z_{R} / L_{u}$ is the electron bunch Fresnel number [21]. The analysis is limited to the fundamental harmonic, since higher harmonics are typically suppressed by the bunch form factor $F(\omega)$ of low-energy bunches used for the generation of $\mathrm{THz}$ radiation.

Integral (11) can be solved using the method of stationary phase [22]. The main contribution to (11) comes from the so-called stationary point, in the vicinity of which the total phase $\Psi(z)$ is quasiconstant:

$$
\frac{\mathrm{d}}{\mathrm{dz}}\left[\frac{k r^{2}}{2 R(z)}-\phi(z)+\psi(z)\right]=0 .
$$

One can check that $\psi^{\prime} \sim 1 / \lambda_{u}$ and $\phi^{\prime} \sim\left(k \sigma_{b}^{2} / R\right)^{\prime} \sim 1 / z_{R}$, where the symbol prime stands for the derivative with respect to $z$. Typically, $z_{R} \sim L_{u}$ so that $\phi^{\prime}$ and $\left(k r^{2} / R\right)^{\prime}$ can safely be disregarded and the stationary phase condition reduces to $\psi^{\prime}(z)=0$. Moreover, it turns out that for the right tapering $(k / R)$ is almost constant with $z$. The explicit equation determining stationary points is given by

$$
\xi \equiv \lambda / \lambda_{0}=1+\mathcal{K}^{2} f_{u}^{2}\left(z_{0}\right) / 2 .
$$

The solution to (11) for nondegenerated stationary points $\Psi^{\prime \prime}\left(z_{0}\right) \neq 0$ reads

$$
\begin{aligned}
E_{\omega}(\xi) \approx & \sum_{j} \sqrt{\frac{2 \pi}{\left|\Psi^{\prime \prime}\left(z_{0, j}\right)\right|}} E_{\omega}^{0} g\left(z_{0, j}\right) f_{u}\left(z_{0, j}\right) \\
& \times \exp \left[\frac{-r^{2}}{w^{2}\left(z_{0, j}\right)}+i \Psi\left(z_{0, j}\right)+i \frac{\pi}{4} \operatorname{sgn} \Psi^{\prime \prime}\left(z_{0, j}\right)\right] .
\end{aligned}
$$

The summation is over all stationary points $z_{0, j}$ that are within the integration interval and located from the ends of the interval at a distance greater than the width of the corresponding resonance [23]. The region of validity of (18) and a set of solutions to (11) valid for any wavelength are given in the Appendix. The asymptotic solution (18) is obtained from Eq. (11) by Taylor expanding the phase around point $z_{0}$ up to the second order and integrating only the phase term while the rest is factored out at point $z_{0}$ thanks to its slow dependence on $z$. The derivatives of $\phi$ and $\left(k r^{2} / R\right)$ may be omitted, since $\phi^{\prime \prime} \sim\left(k \sigma_{b}^{2} / R\right)^{\prime \prime} \sim \psi^{\prime \prime} / N_{u}$. Note that the diffraction effect is accounted for in (18) through $g\left(z_{0, j}\right)$ and $R\left(z_{0, j}\right)$.

\section{OPTIMAL TAPERING}

For a symmetric undulator profile $f_{u}(z)$, condition (17) is satisfied for two positions in the undulator, and the spectral component (18) is the sum of two waves with the same frequency and amplitude but different phases. As is pointed out in Ref. [18], such a superposition results in oscillations in the spectrum of emitted radiation. In order to avoid these oscillations, the undulator profile must be a monotonic function of $z$.

In order for the superposition of the impulses (18) emitted by individual bunches to form the proper quasihalf-cycle pulse not only on axis but also off axis, the waist and curvature of the wavefront along the impulse must be constant. Then, the 1D result (3) applies directly. It turns out that for small $z$ and $f_{u}$ these conditions are roughly fulfilled if $f_{u}(z) \propto z$. Figure 3 shows an example of the change of $g(z)$ and $\left[k(z) \sigma_{b}^{2} / 2 R(z)\right]$ along the undulator for a linear positive taper $f_{u}=z / L_{u}$ and a negative taper $f_{u}=\left(1-z / L_{u}\right)$. For the positive taper, the diffraction effect is mitigated, which has a simple physical interpretation: A short-wavelength part of the impulse generated at the beginning of the undulator (17) has a bigger Rayleigh

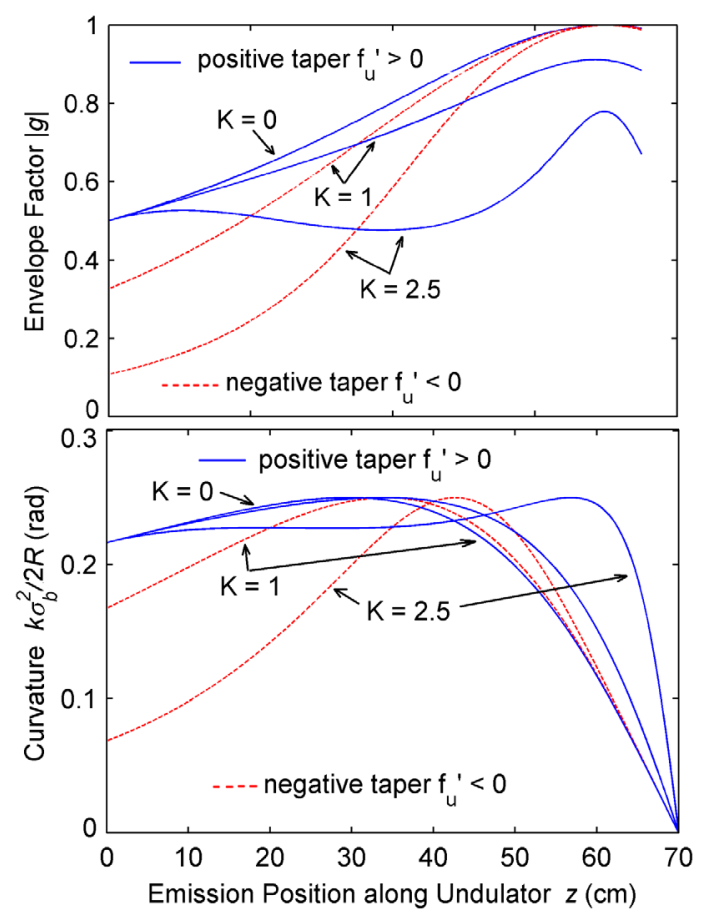

FIG. 3. Typical variation of $g(z)$ and $k(z) \sigma_{b}^{2} / 2 R(z)$ along the undulator for two trial tapers $f_{u}=z / L_{u}$ and $f_{u}=\left(1-z / L_{u}\right)$ referred to as the positive and negative ones. The bunch and undulator parameters are discussed in detail in Sec. V. 
length $z_{R}$ (smaller divergence) than a long-wavelength part generated towards the end of the undulator so that the divergence times the distance is roughly constant. Hence, we limit the following analysis of the sought taper to the positive one $f_{u}^{\prime}>0$, focus the attention on the on-axis radiation, and assume $g(z)=$ const.

The amplitude condition for the generation of a fewcycle pulse [see Eq. (3) and the discussion there] can be met if the spectral density of undulator radiation $\left|E_{\omega}\right|^{2}$ is equal to that of the desired pulse. Hence, by equating $\left|E_{\omega}\right|^{2}$ of (11) to $\left|f_{\omega}\right|^{2}$ of (2) (times some normalization constant) and solving the resulting equation, we can find the optimal undulator profile. But before proceeding to the actual calculation, let us see how the typical spectrum from a strongly tapered undulator compares to the spectra of fewcycle pulses. The comparison is graphically presented in Fig. 4, in which the spectra of the undulator impulse, single-cycle, and 1.5-cycle pulses are shown. To illustrate the features and shape of the undulator spectral distribution, we consider a simple undulator field profile comprising a linear positive taper followed by a quick quadratic decrease. The wavelength for each spectrum is normalized to the value corresponding to the peak of the spectrum so that all the spectral peaks are aligned. The short-wavelength part of the undulator spectrum matches well that of the single-cycle and 1.5-cycle pulses. However, the longwavelength part of these pulses extends much beyond that of the undulator spectrum. Specifically, the upper value of $\xi_{1 / 2}$, defined as the normalized wavelength corresponding to half of the maximum value of the spectral distribution, reaches around 2 and 3 for the single-cycle and 1.5-cycle pulses, respectively. For the undulator spectrum, $\xi_{1 / 2}$

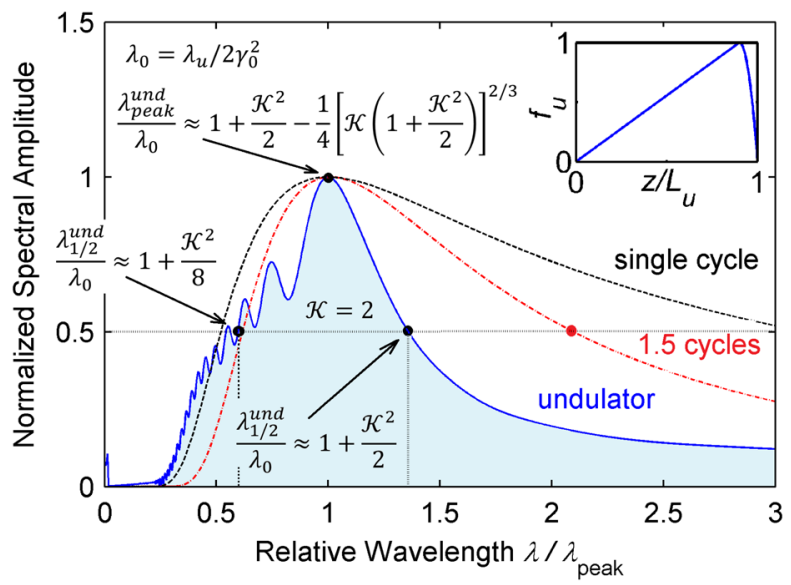

FIG. 4. Comparison of the spectra of the single-cycle and 1.5 -cycle pulses to the on-axis undulator spectrum. The undulator profile is depicted in the inset. The undulator spectrum is calculated numerically using (11) but neglecting diffraction. The wavelength for each spectrum is normalized in the way that the peaks of the spectra are aligned. The wavelengths corresponding to the peak value of the undulator spectrum

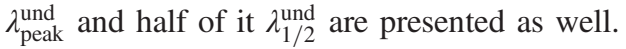

reaches 1.3 at most for any $\mathcal{K}$ of the considered example of the undulator profile. From the results of the Appendix, it follows that in the vicinity of the maximum of $f_{u}$ the spectral amplitude $E_{\omega}(\xi)$ is only half of the result (18) [24] so that $\xi_{1 / 2} \approx 1+\mathcal{K}^{2} / 2\left(\max \left[f_{u}\right]=1\right)$. The peak of the undulator spectrum is located away from $\xi_{1 / 2}$ by the width of the resonance (A9), which approximately reads as

$$
\xi_{\text {peak }} \approx 1+\frac{\mathcal{K}^{2}}{2}-\frac{1}{4}\left[\mathcal{K}\left(1+\frac{\mathcal{K}^{2}}{2}\right)\right]^{2 / 3} .
$$

The bottom value of $\xi_{1 / 2}$ corresponds to $f_{u} \approx 1 / 2$, since $E_{\omega} \propto f_{u}$ so that $\xi_{1 / 2} \approx 1+\mathcal{K}^{2} / 8$.

Because of the discussed difference in the width of the spectrum, it does not seem feasible to generate single-cycle or shorter pulses. In contrast, the quasi-half-cycle pulse (1) has a 50\% narrower spectrum, and it is plausible that such pulses can be produced with a tapered undulator. Below, we derive a closed-form analytical solution for $f_{u}(z)$ yielding the quasi-half-cycle pulses (1) using a simple quadratic approximation to the spectrum (2).

Let us first Taylor expand $\left|f_{\omega}\right|^{2}$ [Eq. (2)] in the vicinity of its maximum. It is convenient to Taylor expand $\left|f_{\omega}\right|^{2}$ with respect to the wavelength, since it is directly proportional to $f_{u}$. The result reads

$$
\left|f_{\omega}\right|^{2} \approx \frac{4}{e^{2}}\left[1-\alpha(\xi-\bar{\xi})^{2}\right],
$$

where, in order to make the coming results more general, we introduced the parameters $\alpha$ and $\bar{\xi}$ being proportional to the inverse normalized bandwidth and peak normalized wavelength, respectively. In the particular case of quasihalf-cycle pulses, $\alpha$ and $\bar{\xi}$ are equal to $8 / p_{0}^{2}$ and $p_{0} / \sqrt{2}$, respectively, with $p_{0}$ being $2 \pi c \sigma_{t} / \lambda_{0}$. Hence, the relation $\alpha \bar{\xi}^{2}=4$ must hold.

The condition $\left|E_{\omega} / E_{\omega}^{0}\right|^{2}=\left|f_{\omega}\right|^{2}$ takes the form

$$
\frac{f_{u} \xi}{f_{u}^{\prime}}=\zeta L_{u}\left[1-\alpha(\xi-\bar{\xi})^{2}\right],
$$

where $\zeta$ is the length normalization parameter. This is the governing equation for the sought optimal undulator profile $f_{u}$. Replacing $\xi$ by $1+f_{u}^{2} \mathcal{K}^{2} / 2$ in accordance with (17), separating the variables $z$ and $f_{u}$, and integrating the quadratures, one obtains

$$
\begin{aligned}
\frac{z}{\zeta L_{u}}= & {\left[\frac{\alpha \bar{\xi}^{2}-1}{2} \log \left(2+f^{2} \mathcal{K}^{2}\right)-\alpha f^{2} \mathcal{K}^{2} / 4\right.} \\
& \left.-\left[\alpha(1-\bar{\xi})^{2}-1\right] \log f\right]\left.\right|_{f=0} ^{f=f_{u}} .
\end{aligned}
$$

The last term is singular at the bottom limit, so we impose the constraint 


$$
\left[\alpha(1-\bar{\xi})^{2}-1\right]=0
$$

which sets a limitation on the profile of spectrum that can be generated with the tapered undulator. Then, the sought solution for the optimum undulator profile reads

$$
\frac{z}{\zeta L_{u}}=\frac{1}{2} \frac{2 \bar{\xi}-1}{(\bar{\xi}-1)^{2}} \log \left[1+\frac{f_{u}^{2} \mathcal{K}^{2}}{2}\right]-\frac{f_{u}^{2} \mathcal{K}^{2}}{4(\bar{\xi}-1)^{2}}
$$

The right-hand side of (24) must be a single-valued function of $f_{u}$, which sets the maximum $f_{u} \mathcal{K}$ for given $\bar{\xi}$ as follows:

$$
\max \left[f_{u} \mathcal{K}\right]=2 \sqrt{\bar{\xi}-1}
$$

This condition limits the bandwidth of generated pulses, since $\bar{\xi}$ and $\alpha$ are interconnected. The normalized duration of the pulse $\bar{\xi}=\sqrt{2} \pi c \sigma_{t} / \lambda_{0}$ is a free parameter within the specified constraints (23) and (25). The normalization parameter $\zeta$ can be found from the condition that $z=L_{u}$ when $f_{u}$ attains its maximum value. Hence,

$$
\zeta=\left[\frac{1}{2} \frac{2 \bar{\xi}-1}{(\bar{\xi}-1)^{2}} \log (2 \bar{\xi}-1)-\frac{1}{\bar{\xi}-1}\right]^{-1} .
$$

Consider the case of the quasi-half-cycle pulses (1). Combining the condition $\alpha \bar{\xi}^{2}=4$ with (23), we obtain that $\bar{\xi}=2$ and $\alpha=2$. Then, from (25) it follows that $\mathcal{K}=2$. The optimal undulator profile, depicted in Fig. 5, can approximately be cast in the form

$$
f_{u}(z) \approx 5.4\left(\frac{\zeta z}{L_{u}}\right)^{3}+10\left(\frac{\zeta z}{L_{u}}\right)^{2}+4.8\left(\frac{\zeta z}{L_{u}}\right)
$$

where $\zeta=0.65$.

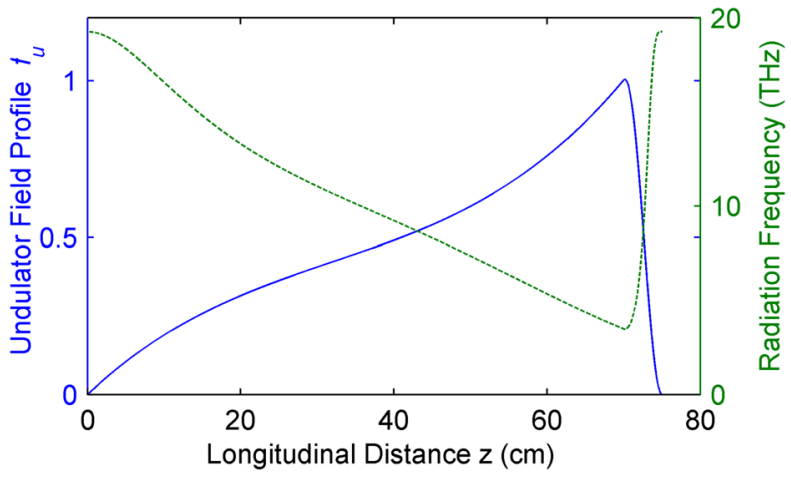

FIG. 5. Optimal undulator field envelope and radiation frequency vs distance in the undulator. $L_{u}=70 \mathrm{~cm}$.

\section{NUMERICAL RESULTS AND DISCUSSION}

As it follows from the analytical results (see Fig. 3), the diffraction effect is mitigated for a linear positive taper. In order to check the impact of diffraction for the found optimal tapering, which is, in fact, quasilinear, a detailed numerical study was performed with a 3D simulation code. The code solves directly the $3 \mathrm{D}$ wave equation with a source in the form of a collection of charged slices whose shape and dynamics are governed by external magnetic fields and radiation fields. The equations of motion are solved using a second-order leapfrog integration, which is symplectic. A typical number of slices per bunch is 100 . The wave equation is solved in the beam frame using the spectral method [25] (basically using Fourier transforms with respect to time and transverse coordinates and propagating in $z$ ). The frequency range in the simulation spans from zero to about $250 \mathrm{THz}$ with a frequency resolution of around $0.04 \mathrm{THz}$. The corresponding resolution in time is around $2 \mathrm{fs}$. The resolution in the phase space of transverse wave numbers (transverse Fourier space) is $1 \mathrm{~mm}^{-1}$, and the integration step in $z$ is $0.5 \mathrm{~mm}$. The code was benchmarked against the numerical results of Ref. [26] for a regular undulator driven by a short electron bunch creating strong coherent spontaneous emission. A discrepancy between the results of the two codes for the emitted energy is less than $5 \%$. The numerical code was also checked against the analytical results of Saldin, Schneidmiller, and Yurkov [21] for a regular undulator and against (18) for a linear taper.

In order to meet the phase condition [see Eq. (3)] for the generation of the quasi-half-cycle pulse, the distance between the adjacent bunches is varied according to the instantaneous resonant wavelength (17). The distances calculated analytically are further adjusted in numerical simulations and are shown in Fig. 6 along with the resonant wavelength (17). The number of bunches is 13 , and the parameters of the single bunch are as follows: charge $Q_{b}=50 \mathrm{pC}$, duration $\sigma_{T}=25 \mathrm{fs}, 2 \mathrm{~mm} \cdot \operatorname{mrad}$ emittance,

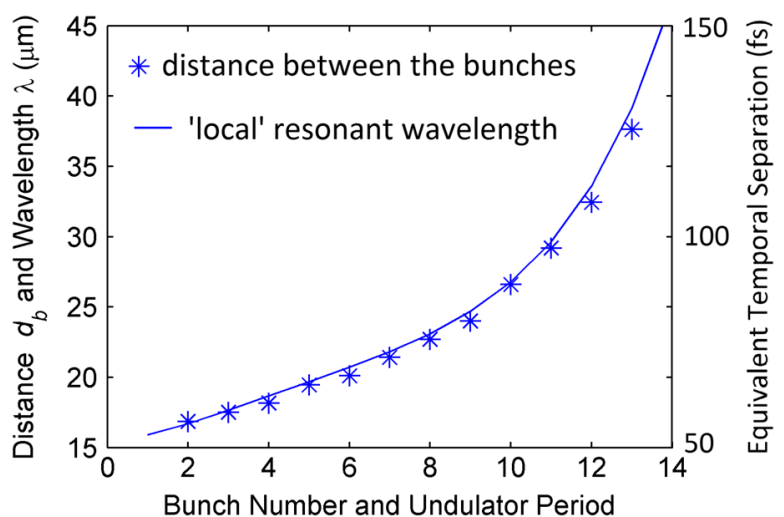

FIG. 6. Distances between the adjacent bunches and the resonant wavelength (17) along the undulator. The corresponding temporal separation between the bunches is also presented. 

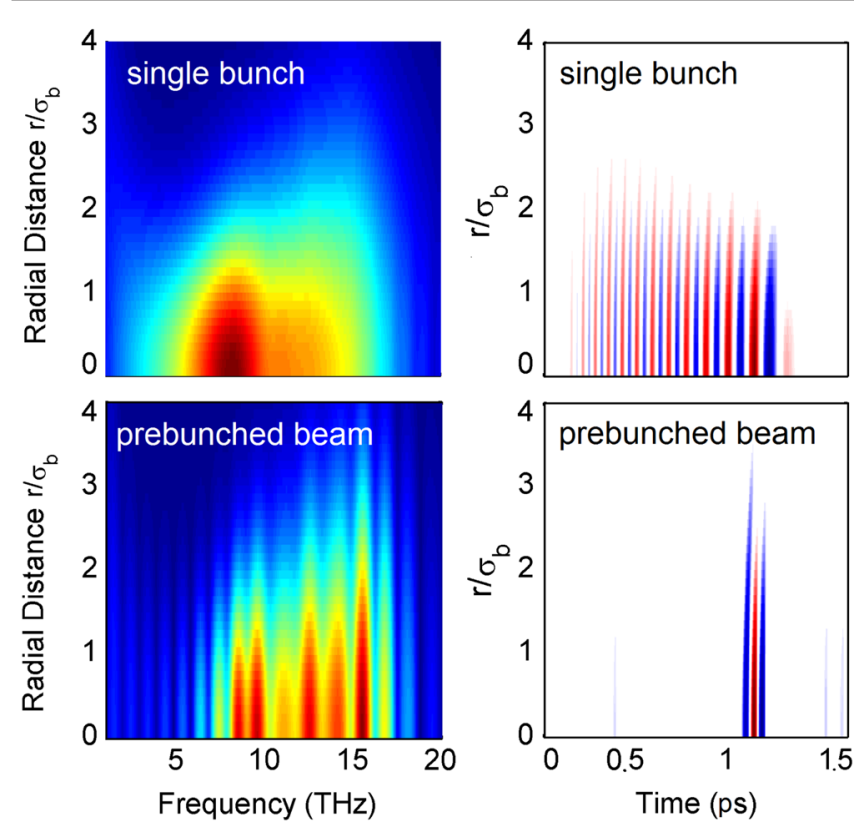

FIG. 7. Top left: Density plot of $E_{\omega}$ generated by a single bunch as a function of the frequency and normalized radial distance $r / \sigma_{b}$; top right: density plot of $E(t)$ generated by a single bunch as a function of the time and $r / \sigma_{b}$; bottom left: $E_{\omega}$ generated by a prebunched beam as a function of the frequency and $r / \sigma_{b}$; bottom right: $E(t)$ generated by a prebunched beam as a function of the time and $r / \sigma_{b}$.

energy $\gamma m_{e} c^{2}=20 \mathrm{MeV}$, and radius $\sigma_{b}=1 \mathrm{~mm}$. There are $N_{u}=15$ undulator periods of $\sigma_{u}=5 \mathrm{~cm}$. The total betatron phase shift $\int_{0}^{L_{u}} \mathrm{~d} z^{\prime} / \lambda_{\beta}\left(z^{\prime}\right)$ is $0.25 \mathrm{rad}$ so that the betatron focusing for such a short undulator can be neglected. The Rayleigh length at the beginning of the undulator $z_{R}=2 \pi \sigma_{b}^{2} / \lambda_{0}=40 \mathrm{~cm}$.

The results of $3 \mathrm{D}$ simulations at the output of the optimally tapered undulator (27) are illustrated in Fig. 7. The density plots of $E_{\omega}$ and $E(t)$ in the frequency-space and time-space domains, respectively, are depicted for a single bunch and a prebunched beam. The on-axis values of $E_{\omega}$ and $E(t)$ are plotted in Fig. 8. From the top left graph in Fig. 8, one can see that the single-bunch undulator spectrum reproduces well the quasi-half-cycle spectrum, which supports the analytical model. Diffraction is mitigated as discussed in Sec. IV. However, if the orientation of the taper is wrong, then the diffraction effect is dramatic and broadband undulator emission is suppressed. To demonstrate this, in Fig. 9, the spectral and temporal distributions of the undulator emission from a single bunch for the reversed optimal tapering are depicted.

The resulting pulse created by the prebunched beam (see bottom right graphs in Figs. 7 and 8) reproduces well the quasi-half-cycle pulse (1) but has long oscillating tails. The contrast of stray peaks to the main peak is around $1: 8$. The peak electric field of the resulting pulse is around $0.1 \mathrm{GV} / \mathrm{m}$, and the pulse energy is about $10 \mu \mathrm{J}$. There are three main factors resulting not in a perfect pulse shape:

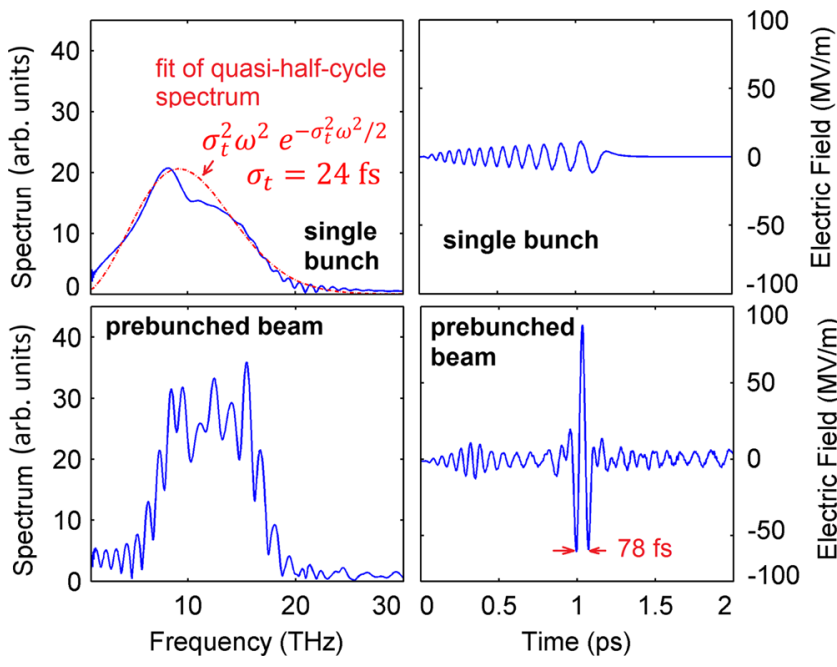

FIG. 8. Top left and top right: On-axis $E_{\omega}$ and $E(t)$ generated by a single bunch vs the frequency and time, respectively; bottom left and bottom right: on-axis $E_{\omega}$ and $E(t)$ generated by a prebunched beam vs the frequency and time, respectively.
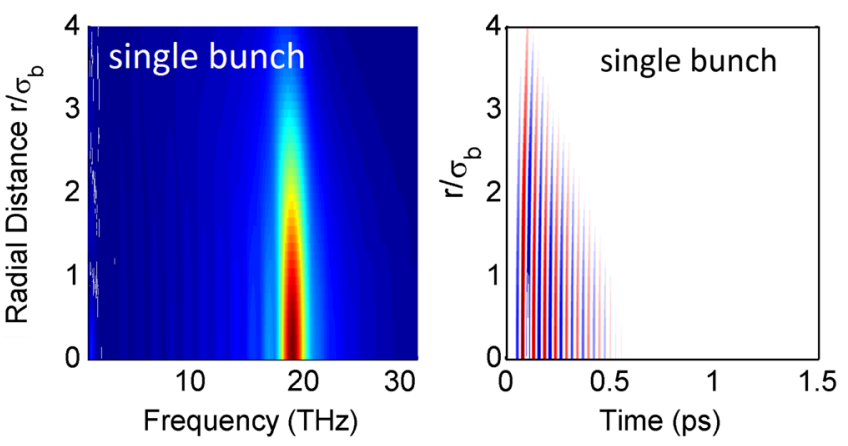

FIG. 9. Spectral and temporal distributions of the undulator emission for the reversed (deliberately wrong) orientation of optimal tapering, $f_{u}^{\mathrm{opt}}\left(L_{u}-z\right)$.

(i) The single-cycle undulator spectrum does not match completely that of the desired pulse; (ii) there are 13 discrete bunches, whereas the phase condition resulting from (3) formally requires an infinite number of radiators (electrons) to compensate for the phase shift of the continuum of frequencies of the chirped undulator impulse; (iii) the contrast is proportional to $1 / N_{\text {bunches }}$ with $N_{u}=N_{\text {bunches }}$, but diffraction limits the maximum length of the undulator. In passing, a genetic numerical optimization algorithm failed to find the undulator profile yielding better results for the single-bunch emission than the analytical profile (27).

\section{SUMMARY AND DISCUSSION}

This paper presents a strategy to generate electromagnetic pulses of the desired spectral bandwidth and temporal shape by using a prebunched electron beam and a tapered undulator. The general formula (18) for the radiation field 
from a heavily tapered undulator taking into account diffraction is derived. It extends the results existing in the literature [27]. An extension of the stationary phase method used to derive (18) is also presented, which can be useful for studies of Compton backscattering [17,18]. By matching the spectrum of the undulator radiation (18) to that of the desired pulse, a generic optimal undulator is found (24). The analytical approach indicates that it is possible to generate pulses that are just one and a half cycles long, but the generation of even shorter pulses does not seem feasible because of the very wide spectrum of such pulses; see Fig. 4. It is interesting to note that diffraction is greatly mitigated for the found optimal tapering, and 3D numerical simulations show that the single-bunch undulator spectrum reproduces well the quasi-half-cycle spectrum.

In order to meet the phase condition of the quasi-halfcycle pulse generation, a train of short electron bunches is used. The distance between the adjacent bunches is varied according to the instantaneous resonant radiation wavelength. The resulting radiation pulse has a peak field of $0.1 \mathrm{GV} / \mathrm{m}$ with a contrast ratio to stray peaks of $8: 1$. The simulation results are presented just at the undulator output. As the generated pulses propagate away from the undulator, the free-space dispersion of transversely confined light will result in the temporal reshaping of the pulses, but it can be reversed by proper focusing [28].

The generation of prebunched beams with the required characteristics has not been demonstrated yet, but the theoretical studies [29] suggest that $100 \mathrm{pC}$ ellipsoidal bunches are compressible to $30 \mathrm{fs}$, and recent experimental results $[30,31]$ give evidence that high-quality ellipsoidal bunches can be produced. Space-charge effects in $200 \mathrm{MV} / \mathrm{m} \mathrm{X}$-band rf guns are greatly reduced. This opens a way for producing prebunched beams by illuminating the photocathode of an rf gun by a train of laser pulses [32].

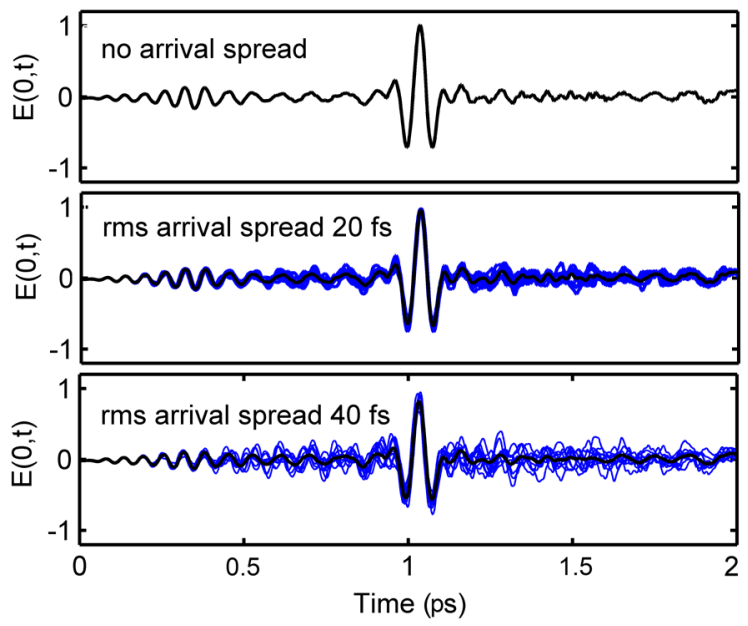

FIG. 10. On-axis $E(t)$ vs the time taking into account the spread of the arrival time of the bunches. The results for ten shots and the averaged value are depicted.
Another option to produce such beams might be nonlinear longitudinal space-charge bunching that naturally results in the increasing period between the bunches because of the rf gun field curvature [33]. The generation of quasi-half-cycle pulses by prebunched beams is quite immune to the spread in arrival time of the electron bunches as demonstrated in Fig. 10.

\section{ACKNOWLEDGMENTS}

The author acknowledges the Stockholm-Uppsala FEL Centre and Swedish Research Council (VR) for support. The author is thankful to Dr. Takashi Tanaka and Dr. Yuichiro Kida for fruitful discussions.

\section{APPENDIX: STATIONARY PHASE METHOD}

Consider the integral

$$
I(\xi)=\int_{z_{A}}^{z_{B}} f\left(z^{\prime} ; \xi\right) \mathrm{e}^{i \psi\left(z^{\prime} ; \xi\right)} \mathrm{d} z^{\prime}
$$

with a generic undulator profile shown in Fig. 11 defined at the interval from $z_{A}$ to $z_{B}$. Function $f(z)$ is a slow function of $z$ compared to ponderomotive phase $\psi$ (15).
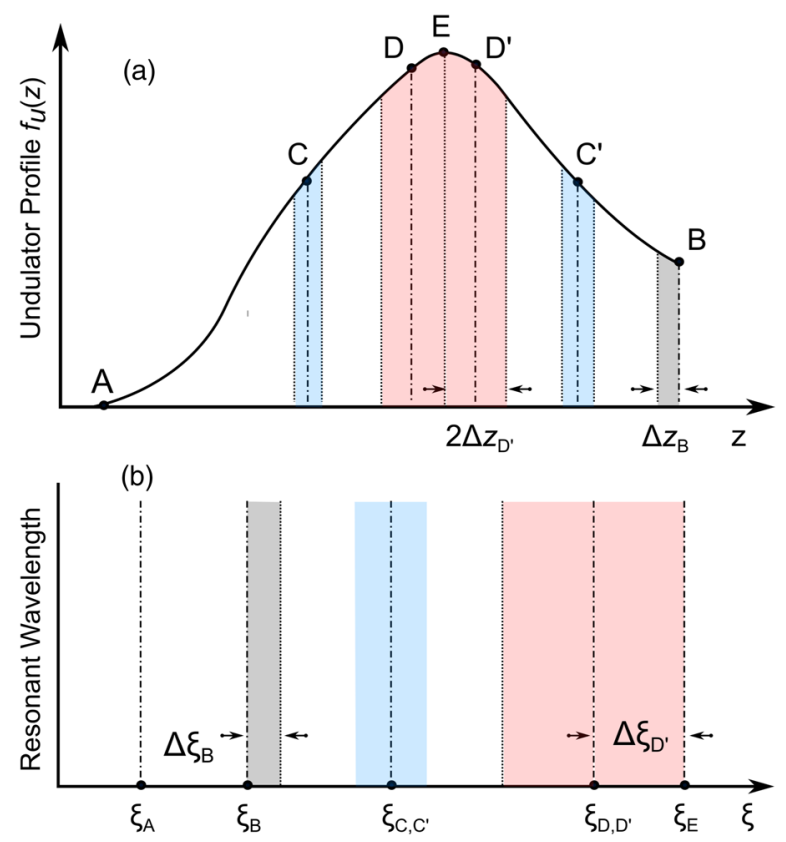

FIG. 11. Generic undulator profile vs longitudinal distance $z$ is schematically depicted in the upper plot. The bottom plot shows corresponding resonant wavelengths (A4) and resonances around them. For $\xi<\xi_{A}$ and $\xi>\xi_{E}$, the solution to (A1) is given by (A2). For wavelengths $\xi$, whose resonances are completely within the integration region and isolated such as the stationary points $C$ and $C^{\prime}$, the solution (A3) is applicable. For the end points $A$ and $B$, the value of the integral is half of (A3). In the vicinity of the degenerated point $E, \psi^{\prime \prime}\left(z_{E}\right)=0$, Eq. (A10) should be used. 
First of all, we notice that in the spectral region characterized by the nonvanishing derivative of the phase, i.e. $\psi^{\prime}(z) \neq 0$, there exists an exact solution to (A1):

$$
I(\xi)=\left.\mathrm{e}^{i \psi(z)} \sum_{j=0}^{\infty} \frac{\mathbf{M}^{j}}{i^{j+1}} \frac{f(z)}{\psi^{\prime}(z)}\right|_{z_{A}} ^{z_{B}}, \quad \mathbf{M}=-\frac{1}{\psi^{\prime}(z)} \frac{\mathrm{d}}{\mathrm{d} z},
$$

which can be derived by integrating by parts. Then, obviously, $\psi^{\prime}(z)=0$ is a special case.

By Taylor expanding $\psi(z)$ to the second order around the stationary point $z_{0}$ such as $\psi^{\prime}\left(z_{0}\right)=0$ and extending the integration limits to infinity, we can integrate (A1) to obtain

$\left.I(\xi) \approx \sum_{j} \sqrt{\frac{2 \pi}{\left|\psi^{\prime \prime}(z)\right|}} f(z) \mathrm{e}^{i \psi(z)+(i \pi / 4) \operatorname{sgn} \psi^{\prime \prime}(z)}\right|_{z=z_{0, j}}$.

The integration region can be extended, because the main contribution to the integral comes from the region over which a variation of $\psi$ is less than $\pi$. Otherwise, if the variation of $\psi$ is greater than $\pi$, then the contributions of the integrand from different points cancel out each other to a large extent, since the factor in front of the exponential is a slow function of $z$ and basically we integrate a sinusoidal function multiplied by a slow-varying amplitude. The condition of "slowness" of $f(z)$ can be formulated as $f(z)$ changes insignificantly over $z_{0} \pm \sqrt{2 \pi / \psi^{\prime \prime}\left(z_{0}\right)}$, which is the scale of the variation of $\psi$ by $\pi$.

Phase $\psi(z)$ vanishes locally for resonant wavelength

$$
\xi_{0}=1+\mathcal{K}^{2} f_{u}^{2}\left(z_{0}\right) / 2,
$$

and solutions to (A4) are limited by $\xi_{\min }=1+\mathcal{K}^{2} \min \left[f_{u}^{2}\right] / 2$ and $\xi_{\max }=1+\mathcal{K}^{2} \max \left[f_{u}^{2}\right] / 2$.

Result (A3) is valid for such wavelengths $\xi_{0}$ that the whole resonance around given $z_{0}\left(\xi_{0}\right)$ is within the integration limits $\left[z_{A}, z_{B}\right]$ as is, for example, the case for the stationary points $z_{C}\left(\xi_{C}\right)$ and $z_{C^{\prime}}\left(\xi_{C^{\prime}}\right)$ depicted in Fig. 11 . For the end points $A$ and $B$, the value of $I(\xi)$ is half of that of the inner point (A3).

If the resonances start to overlap as, for instance, for stationary points $z_{D}\left(\xi_{D}\right)$ and $z_{D^{\prime}}\left(\xi_{D^{\prime}}\right)$, then the value of integration has to be reduced by the amount of overlapping. In the limiting case of the merged resonances corresponding to point $E$, Eq. (18) is not applicable, since the stationary point is degenerated $\psi^{\prime \prime}=0$. The corresponding behavior of the phase is exemplified in Fig. 12.

Let us find the width of resonance $\Delta z$ at the degenerated point $E$ (see Fig. 11) in order to derive a quantitative condition of validity of Eq. (A3). It can be estimated from the condition $\left|\psi\left(z_{0}+\Delta z\right)-\psi\left(z_{0}\right)\right|=\pi$ that yields

$$
\frac{\Delta z}{\lambda_{u}} \approx \frac{\xi / 4}{1-\xi+\mathcal{K}^{2} f_{u}^{2}\left[z_{E}\right] / 2} .
$$

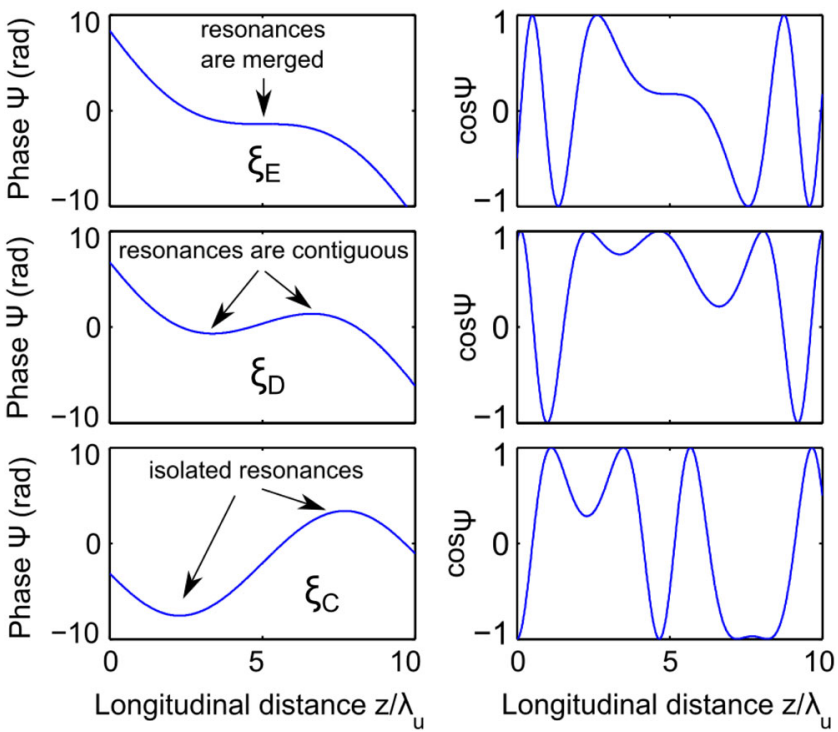

FIG. 12. Illustration of the ponderomotive phase behavior for a parabolic undulator profile $f_{u}=1-\left(2 z / L_{u}-1\right)^{2}$.

The resonance $D^{\prime}$ may be treated as isolated if

$$
z\left(\xi_{D^{\prime}}\right)-\Delta z\left(\xi_{D^{\prime}}\right) \geq z\left(\xi_{E}\right)
$$

The case of equality in this condition gives a critical value of $\xi_{D}^{\prime}$ such that the resonances $D$ and $D^{\prime}$ are contiguous; see the highlighted region $\left[\xi_{D^{\prime}}, \xi_{E}\right]$ in Fig. 11(b) and the corresponding resonances in Fig. 11(a). Let us rewrite Eq. (A6) in the equivalent form

$$
1+\frac{\mathcal{K}^{2}}{2} f_{u}^{2}\left[z\left(\xi_{D}^{\prime}\right)\right]=1+\frac{\mathcal{K}^{2}}{2} f_{u}^{2}\left[z\left(\xi_{E}\right)+\Delta z\left(\xi_{D}^{\prime}\right)\right]
$$

and Taylor expand the right-hand side with respect to $\Delta z$ up to the second order, since $f_{u}^{\prime}\left(z_{E}\right)=0$. It becomes

$$
\xi_{D^{\prime}}=\xi_{E}+\left.\frac{1}{2 !} \frac{\mathcal{K}^{2}}{2} \frac{\mathrm{d}^{2} f_{u}^{2}}{\mathrm{~d} z^{2}}\right|_{z_{E}}\left[\Delta z\left(\xi_{D^{\prime}}\right)\right]^{2}
$$

The left-hand side of Eq. (A8) is equal to that of Eq. (A7) by definition. It is advantageous to seek a solution for $\Delta \xi=\xi_{E}-\xi_{D^{\prime}}$. Then, from Eq. (A5), it follows that

$$
\frac{\Delta z\left(\xi_{D^{\prime}}\right)}{\lambda_{u}} \approx \frac{\xi_{E}}{4 \Delta \xi}
$$

which allows us to derive a cubic equation for $\Delta \xi$ :

$$
\Delta \xi-\frac{\mathcal{K}^{2}}{8}\left|\frac{\mathrm{d}^{2} f_{u}^{2}}{\mathrm{~d} z^{2}}\right|_{z_{E}} \mid\left(\frac{\xi_{E}}{4 \Delta \xi}\right)^{2}=0 .
$$




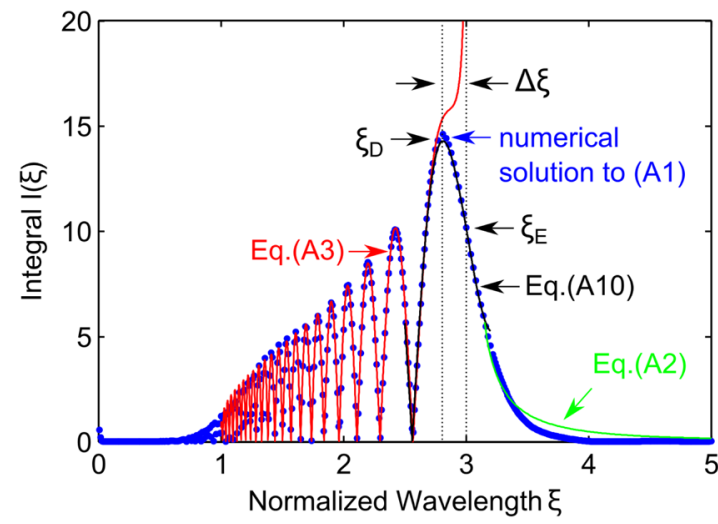

FIG. 13. Comparison of the analytical solutions (A2), (A3), and (A10) to the numerical integration of Eq. (A1).

The spectral width of the resonance is

$$
\Delta \xi=\frac{1}{4} \sqrt[3]{\frac{\mathcal{K}^{2}}{2}\left|\frac{\mathrm{d}^{2} f_{u}^{2}}{\mathrm{~d} z^{2}}\right|_{z_{E}} \mid \xi_{E}^{2}} .
$$

Equation (A9) can also be used to estimate the width of the resonances in the vicinity of the end points $A$ and $B$.

A natural extension of solution (A3) for the degenerated stationary point $z_{E}$ is to Taylor expand $\psi(z)$ up to the third order in the vicinity of $z_{E}$. By using the integral [34]

$$
\int_{0}^{\infty} \cos \left(x^{3} \mp a x\right) \mathrm{x}=\frac{\pi \sqrt{a}}{3 \sqrt{3}}\left\{\begin{array}{c}
J_{-1 / 3}(b)+J_{1 / 3}(b) \\
I_{-1 / 3}(b)-I_{1 / 3}(b)
\end{array}\right\},
$$

where $b=2(a / 3)^{3 / 2}$, we arrive at the solution

$$
\begin{aligned}
I(\xi) \approx & 2 g\left(z_{E}\right) \mathrm{e}^{i \psi\left(z_{E}\right)} \frac{\operatorname{sgn} \psi^{\prime \prime \prime}\left(z_{E}\right)}{\sqrt{3}\left|\psi^{\prime \prime \prime}\left(z_{E}\right)\right| / 6} \frac{\pi \sqrt{a}}{3 \sqrt{3}} \\
& \times\left\{\begin{array}{l}
J_{-1 / 3}(b)+J_{1 / 3}(b), \operatorname{sgn}\left[\psi^{\prime}\left(z_{E}\right) \psi^{\prime \prime \prime}\left(z_{E}\right)\right]<0 \\
I_{-1 / 3}(b)-I_{1 / 3}(b), \operatorname{sgn}\left[\psi^{\prime}\left(z_{E}\right) \psi^{\prime \prime \prime}\left(z_{E}\right)\right]>0
\end{array}\right\} .
\end{aligned}
$$

Here, $a=\psi^{\prime}\left(z_{E}\right) / \sqrt[3]{\left|\psi^{\prime \prime \prime}\left(z_{E}\right)\right| / 6}$.

Equations (A2), (A3), and (A10) give solutions to integral (A1) for all wavelengths. To illustrate the results, the above analytical results are compared to a numerical solution to (A1) in Fig. 13 for a parabolic undulator profile $f_{u}=1-\left(2 z / L_{u}-1\right)^{2}$. The considered undulator profile gives rise to a complicated spectral profile accurately reproduced according to the analytical formulas.

[1] C. Fattinger and D. Grischkowsky, Terahertz beams, Appl. Phys. Lett. 54, 490 (1989).

[2] A. Nahata, D. H. Auston, T. F. Heinz, and C. Wu, Coherent detection of freely propagating terahertz radiation by electro-optic sampling, Appl. Phys. Lett. 68, 150 (1996).

[3] A. Nahata and T. F. Heinz, Reshaping of freely propagating terahertz pulses by diffraction, IEEE J. Quantum Electron. 2, 701 (1996).

[4] D. Giovannini, J. Romero, V. Potoček, G. Ferenczi, F. Speirits, S. M. Barnett, D. Faccio, and M. J. Padgett, Spatially structured photons that travel in free space slower than the speed of light, Science 347, 857 (2015).

[5] S. Feng, H. G. Winful, and R. W. Hellwarth, Opt. Lett. 23, 385 (1998).

[6] T. Kampfrath, K. Tanaka, and K. A. Nelson, Resonant and nonresonant control over matter and light by intense terahertz transients, Nat. Photonics 7, 680 (2013).

[7] D. Fausti, R. I. Tobey, N. Dean, S. Kaiser, A. Dienst, M. C. Hoffmann, S. Pyon, T. Takayama, H. Takagi, and A. Cavalleri, Science 331, 189 (2011).

[8] A. Dienst, M. C. Hoffmann, D. Fausti, J. C. Petersen, S. Pyon, T. Takayama, H. Takagi, and A. Cavalleri, Bi-directional ultrafast electric-field gating of interlayer charge transport in a cuprate superconductor, Nat. Photonics 5, 485 (2011).

[9] K. Yeh, M. Hoffmann, J. Hebling, and K. A. Nelson, Generation of $10 \mu \mathrm{J}$ ultrashort terahertz pulses by optical rectification, Appl. Phys. Lett. 90, 171121 (2007).

[10] J. A. Fülöp, L. Pálfalvi, S. Klingebiel, G. Almási, F. Krausz, S. Karsch, and J. Hebling, Generation of sub$\mathrm{mJ}$ terahertz pulses by optical rectification, Opt. Lett. 37, 557 (2012).

[11] M. Shalaby and C. P. Hauri, Demonstration of a lowfrequency three-dimensional terahertz bullet with extreme brightness, Nat. Commun. 6, 5976 (2015).

[12] M. C. Hoffmann, S. Schulz, S. Wesch, S. Wunderlich, A. Cavalleri, and B. Schmidt, Coherent single-cycle pulses with $\mathrm{MV} / \mathrm{cm}$ field strengths from a relativistic transition radiation light source, Opt. Lett. 36, 4473 (2011).

[13] Z. Wu et al., THz light source at SLAC FACET user facility, in Proceedings of Infrared, Millimeter, and Terahertz Waves (IEEE, New York, 2014), pp. 1-2.

[14] N. A. Vinokurov and Y. U. Jeong, Generating High-Power Short Terahertz Electromagnetic Pulses with a Multifoil Radiator, Phys. Rev. Lett. 110, 064805 (2013).

[15] T. Tanaka, Proposal to Generate an Isolated Monocycle X-Ray Pulse by Counteracting the Slippage Effect in Free-Electron Lasers, Phys. Rev. Lett. 114, 044801 (2015).

[16] Y. Kida, R. Kinjo, and T. Tanaka, Synthesizing high-order harmonics to generate a sub-cycle pulse in free-electron lasers, Appl. Phys. Lett. 109, 151107 (2016).

[17] G. Krafft, Spectral Distributions of Thomson-Scattered Photons from High-Intensity Pulsed Lasers, Phys. Rev. Lett. 92, 204802 (2004).

[18] C. Brau, Oscillations in the spectrum of nonlinear Thomson-backscattered radiation, Phys. Rev. ST Accel. Beams 7, 020701 (2004).

[19] E. Saldin, E. A. Schneidmiller, and M. V. Yurkov, The Physics of Free Electron Lasers (Springer Science, New York, 2000).

[20] D. Alferov, Y. A. Bashmakov, and E. Bessonov, Undulator radiation, Sov. Phys. Tech. Phys. 18, 1336 (1974). 
[21] E. Saldin, E. Schneidmiller, and M. Yurkov, A simple method for the determination of the structure of ultrashort relativistic electron bunches, Nucl. Instrum. Methods Phys. Res., Sect. A 539, 499 (2005).

[22] M. Born and E. Wolf, Principles of Optics (Cambridge University Press, Cambridge, England, 2000).

[23] M. Fedoryuk, Asymptotics: Integrals and Series (Nauka, Moscow, 1987).

[24] Since $\psi$ changes quickly for the decreasing part of $f_{u}$, only half of the resonance around the stationary point contributes to the integral.

[25] W. H. Press, The Art of Scientific Computing, Numerical Recipes 3rd ed. (Cambridge University Press, Cambridge, England, 2007).

[26] V. Z. R. Chulkov and V. Goryashko, Numerical simulation of a super-radiant $\mathrm{THz}$ source driven by femtosecond electron bunches, in Proceedings of FEL2014, Basel, Switzerland, TUP012 (The Paul Scherrer Institute, Villigen, Switzerland, 2014).

[27] A. Gover, R. Ianconescu, A. Friedman, C. Emma, and P. Musumeci, Nucl. Instrum. Methods Phys. Res., Sect. A (to be published).

[28] S. Feng, H. G. Winful, and R. W. Hellwarth, Spatiotemporal evolution of focused single-cycle electromagnetic pulses, Phys. Rev. E 59, 4630 (1999).
[29] S. van der Geer, M. de Loos, T. Van Oudheusden, W. Opt Root, M. van der Wiel, and O. Luiten, Longitudinal phasespace manipulation of ellipsoidal electron bunches in realistic fields, Phys. Rev. ST Accel. Beams 9, 044203 (2006).

[30] P. Piot, Y.-E. Sun, T. Maxwell, J. Ruan, E. Secchi, and J. Thangaraj, Formation and acceleration of uniformly filled ellipsoidal electron bunches obtained via spacecharge-driven expansion from a cesium-telluride photocathode, Phys. Rev. ST Accel. Beams 16, 010102 (2013).

[31] C. Limborg-Deprey et al., Performance of a first generation X-band photoelectron rf gun, Phys. Rev. Accel. Beams 19, 053401 (2016).

[32] L.-X. Yan, J.-F. Hua, Y.-C. Du, Y.-F. Huang, Y. You, D. Wang, W.-Hui Huang, and C.-X. Tang, UV pulse trains by $\alpha$-BBO crystal stacking for the production of THz-rap-rate electron bunches, J. Plasma Phys. 78, 429 (2012).

[33] P. Musumeci, R. Li, and A. Marinelli, Nonlinear Longitudinal Space Charge Oscillations in Relativistic Electron Beams, Phys. Rev. Lett. 106, 184801 (2011).

[34] A. Prudnikov, Y. A. Brychkov, and O. I. Marichev, Integrals and Series: Special Functions (CRC Press, Boca Raton, FL, 1998), Vol. 2. 